\title{
A Study of Hospital Acquired Bacterial Infections and its Antimicrobial Susceptibility Pattern in a Teaching Hospital of Gujarat, India
}

\author{
Dipak M. Panjwani ${ }^{1,2^{*}}$, Sucheta J. Lakhani ${ }^{1}$, \\ Sanjay J. Mehta ${ }^{2}$, Kunjan M. Kikani ${ }^{2}$ and KhushbooMadaan ${ }^{2}$ \\ ${ }^{1}$ Department of Microbiology, SBKS Medical Institute and Research Centre, Sumandeep \\ Vidyapeeth, Piparia (Gujarat), India \\ ${ }^{2}$ Deptartment of Microbiology, C. U. Shah Medical College, Surendranagar (Gujarat), India \\ *Corresponding author
}

\section{Keyw ords \\ Nosocomial infection, Hospital acquired infection, Prevalence and Antimicrobial susceptibility pattern}

\section{Article Info}

Accepted:

08 January 2020

Available Online:

10 February 2020

\section{A B S T R A C T}

Hospital acquired infections (HAI) are counted as major reasons of mortality, morbidity and emotional stress in hospitalized patients. These infections has also enhanced burden on health care institutions. It has also been estimated that at any time over 1.4 million people worldwide suffer from nosocomial infections. The main reason for this alarming situation is resistant microorganisms of hospitals. The objective of this study was to isolate and identify the bacterial etiological agents responsible for nosocomial infection and to evaluate antimicrobial susceptibility pattern of these isolates. This study was conducted in the Department of Microbiology, C. U. Shah Medical College, Surendranagar; Gujarat. Various clinical specimens fulfilling the requisite criteria were included in the study. Specimens were collected and further processed for identification and antimicrobial susceptibility pattern through automated system (Vitek-2) as per standard guidelines. Out of total 1324 culture positive specimens, 187 were classified as HAI as per standard definition. The most frequent type of HAI was surgical site infection (45\%) followed by Respiratory tract infection (25\%). More than $80 \%$ of these types of infections were caused by the Gram-negative Bacteria (GNB). Klebsiella sp. (25\%), Acinetobacter baumannii (19\%), Pseudomonas sp. (16\%), E. coli (13\%) and Staphylococcus aureus (12\%) were the common organisms. Among 24 S. aureus isolates, 18 (78\%) were found to be Methicillin resistance Staphylococcus aureus (MRSA). Majority of Gram-negative isolates showed high resistant against amoxicillin, cefuroxime, cefotaxime and ceftriaxone. The present study reports 187 isolates with HAI. Hand washing practices, operational theater surveillances and periodic training of health care workers can reduce these incidences in

\section{Introduction}

Nosocomial infections also known as hospital-acquired infections are those infections acquired in hospital or healthcare service unit that first appear 48 hours or more after hospital admission within 30 days after discharge following in patient care. They are unrelated to the original illness that brings patients to the hospital and neither present nor incubating at the time of admission. There are some reasons why these infections are even 
more alarming in this era. These include hospital related large number of people who are sick and whose immune system are often in a weak state are more prone to nosocomial infections. The routine use of anti-microbial agents in hospital creates selection pressure for the emergence of the resistant strains of microorganisms. (Revelas, 2012)

Nosocomial infections occur in patients while in hospital or other clinical care facility. Besides harming patients, hospital acquired infections can also affect nurses, doctors, laboratory persons, health care workers and visitors. According to CDC about $10 \%$ of all hospital patients acquire nosocomial infections.

Thus HAI represent a significant proportion of all infectious disease acquired by human. Nosocomial infections have proven to be the most common cause of increased morbidity, mortality, and the cost of the hospitalized patients more so in the intensive care units (ICUs). The increased length of stay for infected patients is the greatest contributor to cost (Willey et al., 2011; Ducel et al., 2012).

The most frequent nosocomial infections are infections of surgical wounds, urinary tract infections and lower respiratory tract infections. The WHO study, and others, has also shown that the highest prevalence of nosocomial infections occurs in intensive care units and in acute surgical and orthopaedic wards. Infection rates are higher among patients with increased susceptibility because of old age, underlying disease, or chemotherapy (Ducel et al., 2012).

Hospitals, and particularly intensive care units, are an important breeding ground for the development and spread of antibiotic resistant bacteria. Increasing incidence of hospital acquired infections caused by antibiotic resistant pathogens is the selection of resistant mutant strains from the patient's own flora during antibiotic treatment. Strategies to control antibiotic resistance in hospitals include multidisciplinary cooperation in implementing local policies on use of antibiotics and infection control measures, timely detection and reporting of the antibiotic resistant strains, improved surveillance, and aggressive control of transmission of epidemic resistant bacteria. Despite of knowing these strategies to control HAI, these infections are still present and are big reason to worry (Struelens, 1998).

\section{Materials and Methods}

This study was conducted in Department of Microbiology, C. U. Shah Medical college, Surendranagar; Gujarat. Various clinical specimens were received in Department of Microbiology and were included in this study during January 2018 to March 2019. Specimens were collected and further processed for identification and antimicrobial susceptibility pattern as per standard guidelines (Ducel et al., 2012).

Specimen were processed for gram stain, cultured on Blood agar and MacConkey agar and incubated at $35^{\circ} \mathrm{c}$ for overnight as per standard guidelines.(Forbes et al., 2007) For inoculation of blood and other sterile body fluids BacT/Alert automation blood culture system was used.

Pathogens were preliminary identified by colony characters and Gram staining. Further identification of organisms was carried out by Vitek 2 automated system. Antimicrobial susceptibility testing was performed by Vitek2 automation system as per latest CLSI guidelines (Weinstein, 2018).

\section{Results and Discussion}

Total 1324 culture positive samples were collected from indoor patients during January 2018 to March 2019. A total of 187 were 
identified as HAI as per standard definition. Out of 187 isolates, $85(45 \%)$ were from surgical site infection, 46 (25\%) were from respiratory site infection, $28(15 \%)$ were from blood stream infection and $28(15 \%)$ from urinary tract infection (Table -1$)$.

Maximum strains of HAI were isolated from Surgery ward $41(22 \%)$. Out of 187 isolates $36(19 \%)$ were from medicine ward, 35 (18\%) from orthopedic ward, $29(15 \%)$ were from MICU and 22 (12\%) from Pediatric ward. Remaining 24 (14\%) isolates obtained from other wards and ICUs. (Table -2$)$

Organism wise distribution of the isolates was also studied. Klebsiella sp. was the most common isolate, having incidence of 51 ( 25 $\%$ ), followed by Acinetobacter baumannii 40 (19\%) and Pseudomonas sp. 33 (16\%). While E. coli and Staphylococcus aureus both having incidence of $26(13 \%)$ and 24 (12\%) respectively. (Table -3$)$

According to antibiotic susceptibility of the Gram positive isolates, all the isolates were susceptible to Vancomycin. S. aureus showed maximum sensitivity against Linezolid $91 \%$, Teicoplanin $91 \%$ and Gentamicin $83 \%$. While S. aureus had low sensitivity rates against Amoxicillin 9\%, Piperacillin/Tazobactam 17\%, Erythromycin $17 \%$ and Imipenem $22 \%$. Among $24(100 \%)$ S. aureus isolates, 18 $(78 \%)$ were found as MRSA. All Enterococcus sp. had shown completely resistance against Ciprofloxacin, Levofloxacin, Erythromycin, Clindamycin and Doxycycline. (Table -4)

According to antibiotic susceptibility of Gram negative lactose fermenter isolates, Klebsiella sp. showed maximum sensitivity against Colistin 94\%, Tigecycline 69\% and Imipenem $57 \%$. While it had low sensitivity rates against Amoxicillin 2\%, Cefuroxime 12\%, Amoxicillin/Clavulanic acid 18\%,
Cefotaxime 18\% and Ceftriaxone 18\%. E. coli isolates were shown $100 \%$ sensitivity against colistin. While it showed least sensitive rates against Amoxicillin 4\%, Cefuroxime 12\%, Cefotaxime $12 \%$ and Ceftriaxone $12 \%$. (Table - 5)

According to antibiotic susceptibility of Gram negative lactose non fermenter isolates, Acinetobacter baumannii showed maximum sensitivity against colistin 95\%, Tigecycline 68\% and Minocycline 36\%. While it showed low sensitivity rates against Imipenem $18 \%$, Piperacilin/Tazobactam 18\%, Cefepime 18\% and Gentamicin 21\%. Pseudomonas sp. showed maximum sensitivity rates against carbapenems group of antibiotics i.e. 55\%. Remaining all Pseudomonas isolates showed less than $50 \%$ sensitivity rates against all antibiotics used in this panel. (Table -6)

Hospital acquired infections are considered as major causes of mortality, enhanced morbidity and emotional stress in hospitalized patients. They also account for significant economic loss and additional burden on health care institutions. It has been estimated that at any time over 1.4 million people worldwide suffer from infectious complications acquired in hospital. The infections acquired in the hospitals may be due to resistant organisms that further accentuate the problem. (Ducel et al., 2002)

In present study maximum numbers of isolates obtained from surgical site infection i.e. $47 \%$ which is different from other studies reported. However high prevalence of hospital acquired urinary infection has reported about $24 \%$ in both S Habibi et al., (2008) and US Kamat et al., (2008). In this study more than $80 \%$ of these types of infections were caused by the Gram-negative Bacteria (GNB). Klebsiella sp. Pseudomonas sp., E. coli, and Acinetobacter baumannii together counted more than $70 \%$ of the 
isolates. Klebsiella sp. was the most common isolate in present study. Similar findings were also reported by US Kamat et al., (2008). Such alarming numbers of GNB in hospital acquired infections has been commented by many investigators.

According to study of N. Akhtar(2010) sensitivity of various gram negative lactose fermenter isolates was tested, Klebsiella showed $79.2 \%$ sensitive against Imipenem, 70.8\% sensitive against Amikacin, 37.5\% sensitive against Ceftriaxone and $16.7 \%$ sensitive against Amoxicillin/Clavulanic acid.

However present study reported sensitivity of Klebsiella, 57\% sensitive against Imipenem, 53\% sensitive against Amikacin, 18\% sensitive against Ceftriaxone and Amoxicillin/ Clavulanic acid each.

Colistin sensitivity rate for Acinetobacter baumannii was maximum among the panel. Apart from Tigecycline all Acinetobacter isolates showed less than $50 \%$ sensitivity against all antibiotics used in this panel. According to Shalini et al., (2010) Pseudomonas showed maximum sensitivity against Imipenem which was similar to the present study.

In present study $17 \%$ of isolates were Gram positive cocci. All the gram positive isolates were susceptible to Vancomycin which is also lined up by studies of Shalini et al., (2010) and N. Akhtar. (2010) In present study S. aureus showed $78 \%$ methicillin-resistant Staphylococcus aureus (MRSA). According to study of US Kamat et al., (2008) the MRSA is $71 \%$ which is reported similar to present study. However Shalini S. et al., (2010) showed $41 \%$ MRSA which is quite lower than present study.

In the study of N. Akhtar (2010) sensitivity of S. aureus was tested, $68.4 \%$ sensitive against Imipenem, $\quad 15.8 \%$ sensitive against Gentamicin, $10.5 \%$ sensitive against Ciprofloxacin and $42 \%$ sensitive against Amoxicillin/Clavulanic acid.

However present study reported sensitivity of S. aureus, $22 \%$ sensitive against Imipenem, 83\% sensitive against Gentamicin, 39\% sensitive against Ciprofloxacin and $17 \%$ sensitive against Amoxicillin/Clavulanic acid.

Table.1 Type of HAI and specimen wise distribution of isolates

\begin{tabular}{|c|c|c|c|c|}
\hline Type of HAI & Specimens & $\begin{array}{c}\text { No. of isolates } \\
(\mathbf{n = 1 8 7})\end{array}$ & $\begin{array}{c}\text { No. of isolates } \\
(\mathbf{n = 1 8 7})\end{array}$ & Percentage \\
\hline $\begin{array}{c}\text { Surgical site } \\
\text { infection }\end{array}$ & Pus & 50 & 85 & $45 \%$ \\
\hline $\begin{array}{c}\text { Respiratory } \\
\text { infection }\end{array}$ & ET & 35 & & $25 \%$ \\
\hline Sputum & 29 & 46 & \\
\hline Septicemia & BAL & 16 & & $15 \%$ \\
\hline $\begin{array}{c}\text { Urinary tract } \\
\text { infection }\end{array}$ & Urine & 28 & 28 & $15 \%$ \\
\hline Total & - & $\mathbf{1 8 7}$ & 28 & $\mathbf{1 0 0 \%}$ \\
\hline
\end{tabular}


Table.2 Ward wise distributions of isolates

\begin{tabular}{|c|c|c|}
\hline Hospital Location & No. of isolates $(\mathbf{n}=\mathbf{1 8 7})$ & Percentage \\
\hline Surgery Ward & 41 & $22 \%$ \\
\hline Medicine Ward & 36 & $19 \%$ \\
\hline Orthopedic ward & 35 & $18 \%$ \\
\hline MICU & 29 & $15 \%$ \\
\hline Pediatric ward & 22 & $12 \%$ \\
\hline NICU & 7 & $4 \%$ \\
\hline ENT ward & 5 & $3 \%$ \\
\hline SICU & 4 & $2 \%$ \\
\hline OBG ward & 3 & $2 \%$ \\
\hline Pulmonary ward & 2 & $1 \%$ \\
\hline Casualty & 2 & $1 \%$ \\
\hline PICU & 1 & $1 \%$ \\
\hline Total & $\mathbf{1 8 7}$ & $\mathbf{1 0 0 \%}$ \\
\hline
\end{tabular}

Table.3 Organism wise distributions of isolates

\begin{tabular}{|c|c|c|}
\hline Organism & No. of organisms (n=207) & Percentage \\
\hline Klebsiella $s p$. & 51 & $25 \%$ \\
\hline Acinetobacter baumannii & 40 & $19 \%$ \\
\hline Pseudomonas $s p$. & 33 & $16 \%$ \\
\hline E. coli & 26 & $13 \%$ \\
\hline Staphylococcus aureus & 24 & $12 \%$ \\
\hline Pseudomonas aeruginosa & 9 & $4 \%$ \\
\hline CONS & 5 & $2 \%$ \\
\hline Acinetobacter lwoffii & 5 & $2 \%$ \\
\hline Enterococcus faecium & 3 & $1 \%$ \\
\hline Enterococcus faecalis & 3 & $1 \%$ \\
\hline Proteus vulgaris & 2 & $1 \%$ \\
\hline Pseudomonas putida & 2 & $1 \%$ \\
\hline Acinetobacter junnii & 2 & $1 \%$ \\
\hline Enterobacter cloaca complex & 1 & $1 \%$ \\
\hline Morganella morganaii & 1 & $1 \%$ \\
\hline Total & $\mathbf{2 0 7}$ & $\mathbf{1 0 0} \%$ \\
\hline
\end{tabular}


Table.4 Antibiotic sensitivity pattern shown by Gram positive isolates

\begin{tabular}{|c|c|c|c|c|}
\hline Organism & $\begin{array}{l}\text { Staphylococcus } \\
\text { aureus } \\
(\mathbf{n}=\mathbf{2 4})\end{array}$ & $\begin{array}{c}\text { CONS } \\
(n=5)\end{array}$ & $\begin{array}{c}\text { Enterococcus } \\
\text { faecium } \\
(\mathbf{n}=\mathbf{3})\end{array}$ & $\begin{array}{c}\text { Enterococcus } \\
\text { faecalis } \\
(\mathbf{n}=\mathbf{3})\end{array}$ \\
\hline Amoxicillin & $9 \%$ & $20 \%$ & - & - \\
\hline $\begin{array}{c}\text { Amoxicillin/ } \\
\text { Clavulanic acid }\end{array}$ & $17 \%$ & $20 \%$ & - & - \\
\hline Benzylpenicillin & $9 \%$ & $20 \%$ & $0 \%$ & $33 \%$ \\
\hline Cefepime & $22 \%$ & $20 \%$ & - & - \\
\hline Cefotaxime & $22 \%$ & $20 \%$ & - & - \\
\hline Cefoxitin & $26 \%$ & $20 \%$ & - & - \\
\hline Ceftriaxone & $22 \%$ & $20 \%$ & - & - \\
\hline Cefuroxime & $22 \%$ & $20 \%$ & - & - \\
\hline Ciprofloxacin & $39 \%$ & $40 \%$ & $0 \%$ & $0 \%$ \\
\hline Clindamycin & $26 \%$ & $60 \%$ & $0 \%$ & $0 \%$ \\
\hline Deptomycin & $78 \%$ & $80 \%$ & - & - \\
\hline Doxycycline & $78 \%$ & $80 \%$ & $33 \%$ & $0 \%$ \\
\hline Erythromycin & $17 \%$ & $60 \%$ & 0 & $0 \%$ \\
\hline Gentamicin & $83 \%$ & $80 \%$ & - & - \\
\hline Imipenem & $22 \%$ & $60 \%$ & - & - \\
\hline Levofloxacin & $39 \%$ & $60 \%$ & $0 \%$ & $0 \%$ \\
\hline Linezolid & $91 \%$ & $80 \%$ & $33 \%$ & $33 \%$ \\
\hline Meropenem & $22 \%$ & $60 \%$ & - & - \\
\hline Oxacillin & $22 \%$ & $60 \%$ & - & - \\
\hline $\begin{array}{l}\text { Pipercillin/ } \\
\text { Tazobactam }\end{array}$ & $17 \%$ & $20 \%$ & - & - \\
\hline Teicoplanin & $91 \%$ & $80 \%$ & $33 \%$ & $100 \%$ \\
\hline $\begin{array}{l}\text { Trimethoprim/ } \\
\text { Sulfamethoxazole }\end{array}$ & $65 \%$ & $60 \%$ & - & - \\
\hline Vancomycin & $100 \%$ & $100 \%$ & $100 \%$ & $100 \%$ \\
\hline
\end{tabular}

(Percentages are reflected as sensitive rates) 
Table.5 Antibiotic sensitivity pattern shown by Gram negative lactose fermenter isolates

\begin{tabular}{|c|c|c|c|}
\hline $\begin{array}{l}\text { Antibiotic } \\
\text { Organism }\end{array}$ & $\begin{array}{l}\text { Klebsiella sp. } \\
\quad(\mathrm{n}=\mathbf{5 1})\end{array}$ & $\begin{array}{l}\text { E. coli } \\
(n=26)\end{array}$ & $\begin{array}{c}\text { Enterobacter } \\
\text { cloacae complex } \\
(\mathbf{n}=1)\end{array}$ \\
\hline Amikacin & $53 \%$ & $58 \%$ & $0 \%$ \\
\hline Amoxicillin & $2 \%$ & $4 \%$ & $0 \%$ \\
\hline Amoxicillin/Clavulanic acid & $18 \%$ & $15 \%$ & $0 \%$ \\
\hline Ampicillin & $2 \%$ & $4 \%$ & $0 \%$ \\
\hline Cefepime & $24 \%$ & $23 \%$ & $0 \%$ \\
\hline Cefoperazone/Salbactam & $27 \%$ & $31 \%$ & $0 \%$ \\
\hline Cefotaxime & $18 \%$ & $12 \%$ & $0 \%$ \\
\hline Ceftriaxone & $18 \%$ & $12 \%$ & $0 \%$ \\
\hline Cefuroxime & $12 \%$ & $12 \%$ & $0 \%$ \\
\hline Cefuroxime Axetil & $12 \%$ & $12 \%$ & $0 \%$ \\
\hline Ciprofoxacin & $39 \%$ & $15 \%$ & $0 \%$ \\
\hline Colistin & $94 \%$ & $100 \%$ & $100 \%$ \\
\hline Ertapenem & $49 \%$ & $65 \%$ & $0 \%$ \\
\hline Gentamicin & $49 \%$ & $42 \%$ & $0 \%$ \\
\hline Imipenem & $57 \%$ & $69 \%$ & $0 \%$ \\
\hline Meropenem & $53 \%$ & $69 \%$ & $0 \%$ \\
\hline Nalidixic Acid & $29 \%$ & $15 \%$ & $0 \%$ \\
\hline Piperacillin/Tazobactam & $35 \%$ & $35 \%$ & $0 \%$ \\
\hline Tigecycline & $69 \%$ & $73 \%$ & $0 \%$ \\
\hline Trimethoprim/Sulfamethoxazole & $35 \%$ & $31 \%$ & $0 \%$ \\
\hline
\end{tabular}

(Percentages are reflected as sensitive rates) 
Table.6 Antibiotic sensitivity pattern shown by Gram negative lactose non fermenter isolates

\begin{tabular}{|c|c|c|c|c|c|c|c|c|}
\hline Antibiotic & 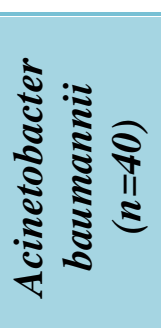 & 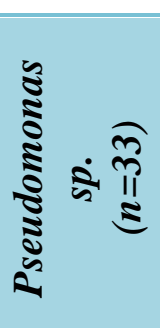 & 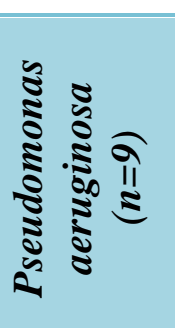 & 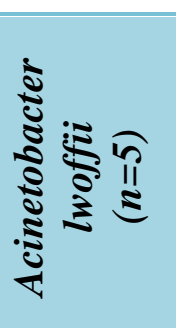 & 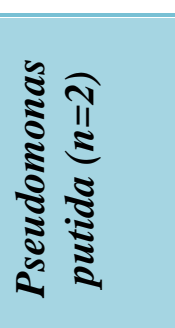 & 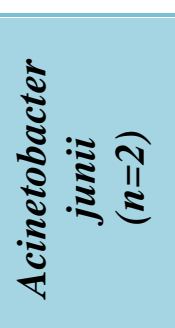 & 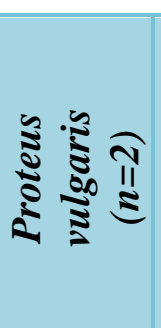 & 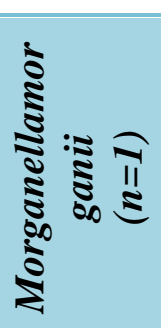 \\
\hline Amikacin & - & $48 \%$ & $56 \%$ & $20 \%$ & $100 \%$ & $50 \%$ & $0 \%$ & $100 \%$ \\
\hline Cefepime & $18 \%$ & $39 \%$ & $56 \%$ & $20 \%$ & $100 \%$ & $100 \%$ & $0 \%$ & $100 \%$ \\
\hline $\begin{array}{l}\text { Cefoparazone/ } \\
\text { Salbactam }\end{array}$ & $18 \%$ & $36 \%$ & $44 \%$ & $60 \%$ & $50 \%$ & $100 \%$ & $0 \%$ & $100 \%$ \\
\hline Ceftazidime & $18 \%$ & $33 \%$ & $56 \%$ & $0 \%$ & $100 \%$ & $50 \%$ & $0 \%$ & $100 \%$ \\
\hline Ciprofloxacin & $18 \%$ & $45 \%$ & $56 \%$ & $40 \%$ & $0 \%$ & $100 \%$ & $0 \%$ & $0 \%$ \\
\hline Colistin & $95 \%$ & - & $100 \%$ & $100 \%$ & - & $100 \%$ & $50 \%$ & $0 \%$ \\
\hline Doripenem & $18 \%$ & $52 \%$ & $56 \%$ & $40 \%$ & - & $50 \%$ & $0 \%$ & $0 \%$ \\
\hline Gentamicin & $21 \%$ & $42 \%$ & $56 \%$ & $40 \%$ & $100 \%$ & $100 \%$ & $0 \%$ & $100 \%$ \\
\hline Imipenem & $18 \%$ & $55 \%$ & $56 \%$ & $40 \%$ & $100 \%$ & $100 \%$ & $0 \%$ & $0 \%$ \\
\hline Levofloxacin & $18 \%$ & $39 \%$ & $67 \%$ & $60 \%$ & 0 & $100 \%$ & $0 \%$ & $0 \%$ \\
\hline Meropenem & $24 \%$ & $55 \%$ & $56 \%$ & $40 \%$ & $100 \%$ & $100 \%$ & $0 \%$ & $100 \%$ \\
\hline Minocycline & $36 \%$ & $45 \%$ & - & $60 \%$ & $100 \%$ & $100 \%$ & $0 \%$ & $0 \%$ \\
\hline $\begin{array}{l}\text { Pipercillin/ } \\
\text { Tazobactam }\end{array}$ & $18 \%$ & $48 \%$ & $44 \%$ & $100 \%$ & $0 \%$ & $100 \%$ & $0 \%$ & $0 \%$ \\
\hline $\begin{array}{c}\text { Ticarcillin/ } \\
\text { Clavulanic Acid }\end{array}$ & $18 \%$ & $36 \%$ & $33 \%$ & $60 \%$ & $0 \%$ & $100 \%$ & $0 \%$ & $0 \%$ \\
\hline Tigecycline & $68 \%$ & $45 \%$ & - & $100 \%$ & $50 \%$ & $100 \%$ & $0 \%$ & $0 \%$ \\
\hline $\begin{array}{c}\text { Trimethoprim/ } \\
\text { Sulfamethoxazole }\end{array}$ & $24 \%$ & $21 \%$ & - & $40 \%$ & $0 \%$ & $100 \%$ & $0 \%$ & $0 \%$ \\
\hline
\end{tabular}

(Percentages are reflected as sensitive rates) 
Incidence of Nosocomial Infections is increasing day by day. The factors that can account for the higher infection rate may be the availability of high number of visitors, lack of knowledge and proper monitoring and setup of the hospital.

This frightening incidence of Nosocomial Infections can be reduced by decontamination and cleaning of soiled instruments and other items. Sterilization and high-level disinfection processes and improving safety in operating rooms and other high-risk areas can also decrease the incidence of Hospital acquired infections. (Ginawi et al., 2014).

The present study on the microbiological profile and antimicrobial susceptibility shows 187 isolates with HAI. The most frequent site of HAI was surgical site followed by respiratory tract. Klebsiella sp., Acinetobacter baumannii and Pseudomonas sp. were common organisms followed by E. coli and Staphylococcus aureus. Maximum isolates were isolated from surgery ward followed by medicine ward.

Gram positive isolates shown most sensitivity towards vancomycin and gram negative isolates towards colistin. The high frequency of HAI and poor sensitivity of common antibiotics suggests the implementation of strict measures regarding infection control. These measures include hand washing practices, operational theater surveillances and periodic training of health care workers.

\section{References}

Revelas A. Healthcare - associated infections: A public health problem. Nigerian Medical Journal. 2012;53(2):59.

Wiley J, Sherwood M, L.M., Woolverton, C. J. Prescott's microbiology. 8th ed. New York: McGraw-Hill Higher Education; 2013.
Ducel G, Fabry J, Nicolle L. Prevention of Hospital Acquired Infections. A practical guide.2nd ed. WHO manual; 2012.

Struelens M, The epidemiology of antimicrobial resistance in hospital acquired infections: problems and possible solutions. BMJ: British Medical Journal. 1998, 317(7159): 652.

Forbes BA, Sahm DF, Weissfeld AS, edts; Bailey and Scott's.Diagnostic microbiology.12th ed. Mosby Elsevier 2007

Weinstein M. Performance standards for antimicrobial susceptibility testing. M100. 28th ed.: Clinical and Laboratory Standards Institute; 2018.

Ducel G, Fabry J, Nicolle L. Guidelines on prevention and control of hospital associated infection. World Health Organization. 2nd ed. New Delhi: WHO Regional Office for South-East Asia; 2002.

Habibi S, Wig N, Agarwal S, Sharma S, Lodha R, Pandey R and Kapil A. Epidemiology of nosocomial infections in medicine intensive care unit at a tertiary care hospital in northern India. Tropical Doctors 2008; 38: 233-5.

Kamat U, Ferreira A, Savio R, Motghare D. Antimicrobial Resistance Among Nosocomial Isolates in a Teaching Hospital in Goa. Indian Journal of Community Medicine. 2008, 33(2): 89-92.

Akhtar N. Hospital Acquired Infections in a Medical Intensive Care Unit. Journal of the College of Physicians and Surgeons Pakistan 2010, 20 (6): 386-90.

Shalini S, Kranthi K, Gopalkrishna K. The Microbiological profile of nosocomial infections in the intensive care unit.Journal of Clinical and Diagnostic Research.2010 Oct, 4:3109-12.

Ginawi I, Mohd. Saleem, Sigh M, Vaish A, Ahmad I, Srivastava V, Fahad A. Hospital Acquired Infections Among 
Patients Admitted in the Medical and Surgical Wards of a Non-Teaching Secondary Care Hospital in Northern
India. Journal of Clinical and Diagnostic Research. 2014, 8(2): 81-3.

\section{How to cite this article:}

Dipak M. Panjwani, Sucheta J. Lakhani, Sanjay J. Mehta, Kunjan M. Kikani and Khushboo Madaan. 2020. A Study of Hospital Acquired Bacterial Infections and its Antimicrobial Susceptibility Pattern in a Teaching Hospital of Gujarat, India. Int.J.Curr.Microbiol.App.Sci. 9(02): 1399-1408. doi: https://doi.org/10.20546/ijcmas.2020.902.162 